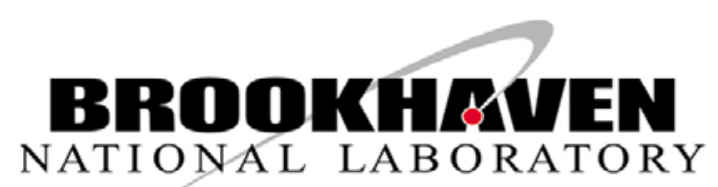

BNL-113428-2017-JA

\title{
Doubling the critical current density in superconducting FeSe0.5Te0.5 thin films by low temperature oxygen annealing
}

\author{
Cheng Zhang, Weidong Si, and Qiang Li \\ Submitted to Applied Physics Letters \\ November 2016 \\ Condensed Matter Physics and Materials Science Department \\ Brookhaven National Laboratory \\ U.S. Department of Energy \\ USDOE Office of Science (SC), \\ Basic Energy Sciences (BES) (SC-22)
}

Notice: This manuscript has been authored by employees of Brookhaven Science Associates, LLC under Contract No. DE- SC0012704 with the U.S. Department of Energy. The publisher by accepting the manuscript for publication acknowledges that the United States Government retains a non-exclusive, paid-up, irrevocable, world-wide license to publish or reproduce the published form of this manuscript, or allow others to do so, for United States Government purposes. 


\section{DISCLAIMER}

This report was prepared as an account of work sponsored by an agency of the United States Government. Neither the United States Government nor any agency thereof, nor any of their employees, nor any of their contractors, subcontractors, or their employees, makes any warranty, express or implied, or assumes any legal liability or responsibility for the accuracy, completeness, or any third party's use or the results of such use of any information, apparatus, product, or process disclosed, or represents that its use would not infringe privately owned rights. Reference herein to any specific commercial product, process, or service by trade name, trademark, manufacturer, or otherwise, does not necessarily constitute or imply its endorsement, recommendation, or favoring by the United States Government or any agency thereof or its contractors or subcontractors. The views and opinions of authors expressed herein do not necessarily state or reflect those of the United States Government or any agency thereof. 

Thin Films by Low Temperature Oxygen Annealing

PACS numbers: 74.70.Dd, 73.20.At, 74.70.-b

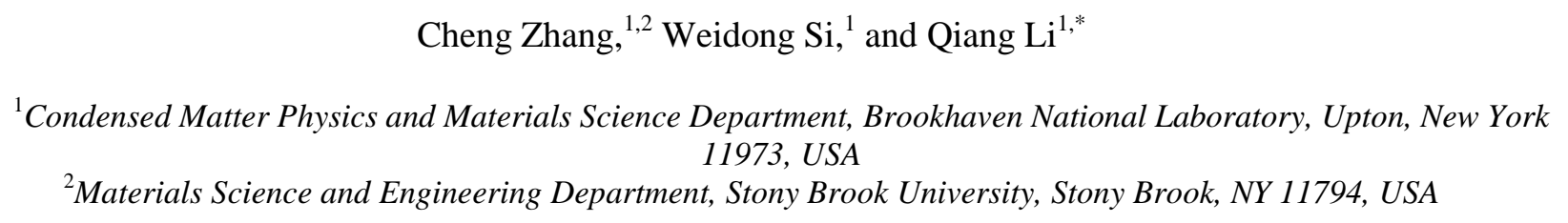

*Corresponding author’s email: qiangli@bnl.gov (Q.L.).

\begin{abstract}
Iron-chalcogenide superconducting thin films and coated conductors are attractive for potential high field applications at liquid helium temperature for their high critical current densities $J_{\mathrm{c}}$,

Abstract low anisotropies, and relatively strong grain couplings. Embedding flux pinning defects is a general approach to increase the in-field performance of superconductors. However, many effective pinning defects can adversely affect the zero field or self-field $J_{c}$, particularly in cuprate high temperature superconductors. Here, we report doubling of the self-field $J_{\mathrm{c}}$ in $\mathrm{FeSe}_{0.5} \mathrm{Te}_{0.5}$ films by low temperature oxygen annealing, reaching $\sim 3 \mathrm{MA} / \mathrm{cm}^{2}$. In-field performance is also dramatically enhanced. Our results demonstrate that low temperature oxygen annealing is a simple and cost-efficient post-treatment technique which can greatly help to accelerate the potential high field applications of the iron-based superconductors.
\end{abstract}


Iron-based superconductors have attracted great interests since their discovery in 2006 by

2 Kamihara et al. ${ }^{1}$ They are superconducting materials with transition temperature, $T_{\mathrm{c}}$, up to $100 \mathrm{~K}$ in

3 monolayer thin films, and very high upper critical field above $100 \mathrm{~T}^{2-8}$ Combined with low

4 anisotropies in magnetic field, this class of superconductors is considered very promising for high

5 field applications. A key limiting factor for the iron based superconductors is the critical current

6 density, $J_{\mathrm{c}}$, that still needs to be raised at or above the level of the competing superconductors.

7 Currently, cuprate high- $T_{\mathrm{c}}$ superconductors (HTS) are the dominating one in terms of the $J_{\mathrm{c}}$

8 performance. ${ }^{7}$ However, the cuprate HTS have intrinsic high anisotropy and low tolerance of grain

9 boundary misalignment. When these drawbacks coupled with high processing cost, cuprate HTS

10 become less competitive than the low temperature $\mathrm{Nb}_{3} \mathrm{Sn}$ in high-magnetic field (up to $14 \mathrm{~T}$ )

11 applications at liquid helium temperature on the cost-performance basis. The recent developments of

12 thin film iron-based superconductors and coated conductors have provided an alternative

13 superconducting material potentially being used in the next generation high field devices. High

14 self-field $J_{\mathrm{c}}$ over $4 \mathrm{MA} / \mathrm{cm}^{2}$ at $4.2 \mathrm{~K}$ has been observed in iron pnictide " 122 " thin films. ${ }^{9-11}$ High

15 self-field $J_{\mathrm{c}}$ above $1 \mathrm{MA} / \mathrm{cm}^{2}$ was also reported in iron chalcogenide films. ${ }^{12,13}$ Most recently, a high

16 field isotropic $J_{c}$ has been observed in $\mathrm{FeSe}_{0.5} \mathrm{Te}_{0.5}$ thin films grown on $\mathrm{CaF}_{2}$ substrates with a

17 self-field $J_{\mathrm{c}}$ over $2 \mathrm{MA} / \mathrm{cm}^{2}$ at $5 \mathrm{~K}^{14}$

18 There are some concerns for the practical application of the iron-based superconductors. For

19 example, the toxic element As in the most studied system, iron pnictides, is one of them, although the

20 superconducting iron pnictides have a relatively higher $T_{\mathrm{C}}$ and $J_{\mathrm{C}}$ among iron-based superconductors.

21 The remaining magnetic excess Fe in the synthesis of the iron based superconductors is another issue,

22 as they can act as the Cooper pair breakers that severely weaken superconductivity. ${ }^{15,16}$ Report has

23 shown that 3\% of excess Fe will totally suppress superconductivity in FeSe, which normally has a $T_{\mathrm{c}}$ 
$1 \sim 8 \mathrm{~K}^{17}$. The sensitivity of the superconducting properties to the excess Fe makes it difficult to

2 produce the reliable iron chalcogenide samples of consistent quality.

Efforts have been made by post-treatment to remove the excess Fe. It has been reported that

4 the excess Fe can be effectively reduced through reaction with acid, oxygen, even alcohol

5 beverages ${ }^{18-26}$. Among these methods, oxygen annealing is found to be most effective.

6 Enhancements on both $T_{\mathrm{c}}$ and $J_{\mathrm{c}}$ were observed in annealed $\mathrm{FeSe}_{1-x} \mathrm{Te}_{x}$ single crystals. ${ }^{21,25,26}$ Yet the

7 study of the annealing effect on thin films of ion-based superconductors, which mostly have higher $T_{\mathrm{c}}$

8 and $J_{\mathrm{c}}$ than those of bulk, is still lacking.

Here we report a strong enhancement of superconducting properties in $\mathrm{FeSe}_{0.5} \mathrm{Te}_{0.5}$ (FST) thin

10 films, through low temperature oxygen annealing. Iron chalcogenides, known as the " 11 " system, are

11 an interesting sub-class of iron-based superconductors. They have the simplest crystal structure. $J_{\mathrm{c}}$

12 anisotropy of the $\mathrm{FeSe}_{1-x} \mathrm{Te}_{x}$ films in magnetic field can be as low as 2, lower than that of pnictides ${ }^{7}$.

13 The optimum superconductivity was found in $\mathrm{FeSe}_{1-x} \mathrm{Te}_{x}$ when $x \cong 0.5$, with a $T_{\mathrm{c}}=14.5 \mathrm{~K}$ in bulk

14 materials. Our previous study shows an enhanced $T_{\mathrm{c}}$ and $J_{\mathrm{c}}$ in FST thin films made by pulsed laser

15 deposition (PLD) ${ }^{12,17}$. With a $\mathrm{CeO}_{2}$ buffer, the films exhibit a self-field $J_{\mathrm{c}}$ above $1 \mathrm{MA} / \mathrm{cm}^{2}$ at $4.2 \mathrm{~K}$. In

16 this letter, we show that low temperature oxygen annealing can enhance $J_{\mathrm{c}}$ dramatically. At $5 \mathrm{~K}$,

17 self-field $J_{\mathrm{c}}$ in the annealed films can reach $3 \mathrm{MA} / \mathrm{cm}^{2}$.

The FST thin films were grown on single crystal substrates by PLD with a $\mathrm{CeO}_{2}$ buffer. The

19 films are of $3 \mathrm{~mm}$ by $5 \mathrm{~mm}$ and the thickness of the films is around $130 \mathrm{~nm}$. The detailed film synthesis

20 has been reported previously ${ }^{12}$. We performed oxygen and vacuum annealing on two sets of films.

21 The oxygen annealing was carried out sequentially at $90^{\circ} \mathrm{C}$ in 100 mTorr oxygen environment in four

22 steps with the annealing time of 15, 30, 60 and 120 minutes. We found that higher oxygen annealing

23 temperature than $90^{\circ} \mathrm{C}$ weakens superconductivity in these films. Vacuum annealing at $1 \times 10^{-8} \mathrm{Torr}$ 
was done at $200^{\circ} \mathrm{C}$ in three steps with the annealing time of 15,30 and 60 minutes. Non-destructive magnetization measurement, with magnetic field perpendicular to the film surface, was performed to characterize the samples after growth and each step of the annealing to compare the changes of superconducting properties. Magnetization measurement is particularly suitable for continuously monitoring the property change. The derived $J_{\mathrm{c}}$ values from the magnetic hysteresis measurement based on the Bean critical state mode ${ }^{28}$ were compared with the $J_{\mathrm{c}}$ values obtained directly from the transport measurement on the laser patterned film bridges, and their differences are within $10 \%$.

Fig. 1(a) shows the magnetic field dependence of $J_{\mathrm{c}}$ at $5 \mathrm{~K}$ for one of the sequentially annealed FST films in oxygen for different annealing time, where magnetic field is applied along the c-axis of the film. Inset shows the magnetization measurement of the films under zero field cool (ZFC) and field cool (FC) for $B=2$ gauss (// c-axis). It is apparent that a short term oxygen annealing remarkably enhances $J_{\mathrm{c}}$ in all fields. The $J_{\mathrm{c}}$ values can be further enhanced by increasing the annealing time before saturated at the last step of two hours annealing - upon which oxygen annealing for longer time does not further enhance the $J_{\mathrm{c}}$. Fully oxygen annealing the film had the self-field $J_{\mathrm{c}}$ value more than doubled, from $1.24 \mathrm{MA} / \mathrm{cm}^{2}$ in the as grown films to $2.54 \mathrm{MA} / \mathrm{cm}^{2}$, as shown in Fig. 1(a). In-field $J_{\mathrm{c}}$ enhancement is much more impressive - more than $300 \%$ increase is achieved at fields above 1.5T. Fig. 1(b) shows the magnetic field dependence of the $J_{\mathrm{c}}$ enhancement, defined as $\frac{J_{c}^{\text {annealed }}-J_{c}^{\text {as-grown }}}{J_{c}^{\text {as-grown }}}$. The process has been repeated in several films, where a similar magnitude of $J_{\mathrm{c}}$ enhancement was observed, with the highest self-field $J_{\mathrm{c}}$ reaching $3 \mathrm{MA} / \mathrm{cm}^{2}$ at $5 \mathrm{~K}$. While $J_{\mathrm{c}}$ is enhanced by oxygen annealing, we notice a small and gradual reduction of $T_{\mathrm{c}}$ as the annealing time increases. This is shown in the inset of Fig. 1(a). After two hours oxygen annealing, the onset $T_{\mathrm{c}}$ reduces from $17.6 \mathrm{~K}$ to $16.2 \mathrm{~K}$, but the transitions becomes sharper.

Fig. 2 shows the magnetic field dependence of $J_{\mathrm{c}}$ and the $J_{\mathrm{c}}$ enhancement at $5 \mathrm{~K}$ for one of 
1 the sequentially annealed FST films in vacuum for different annealing time. The change of $T_{\mathrm{c}}$ of the

2 film after vacuum annealing, shown in the inset, similar to that of oxygen annealed sample. We found

3 the magnitude of $J_{\mathrm{c}}$ enhancement by vacuum annealing saturates at annealing time of $\sim 1-2$ hours, and

4 is much less than that by the oxygen annealing. The self-field $J_{\mathrm{c}}$ of this fully vacuum annealed film is

$51.38 \mathrm{MA} / \mathrm{cm}^{2}, \sim 38 \%$ above that of the as grown films, and in-field $J_{\mathrm{c}}$ enhancement is up to $180 \%$.

6 It is evident that the annealing environment plays a significant role in the film's $J_{\mathrm{c}}$

7 performance. To further demonstrate that oxygen annealing is superior, we gave the last vacuum

8 annealed film (solid triangle in Fig. 2) an additional 2-hour oxygen annealing at $90^{\circ} \mathrm{C}$. The result

9 (open circle) is plotted in Fig. 2 for comparison. Additional oxygen annealing leads to the self-field $J_{\mathrm{c}}$

10 being greatly improved to $2.27 \mathrm{MA} / \mathrm{cm}^{2}$ and again about $300 \%$ enhancement in the in-field $J_{\mathrm{c}}$ on

11 average was observed, which is similar to that of the fully oxygen annealed film (open circles) as seen

12 in Fig. 1. We notice little or no change in $T_{\mathrm{c}}$ after additional oxygen annealing.

Let us discuss the possible origin of the observed $J_{\mathrm{c}}$ enhancement. One of the explanations

14 could be the removal of the excess Fe in the FST films. It is known that in bulk FST, excess Fe atoms

15 are magnetic, and act as the Cooper breakers and suppress superconductivity. ${ }^{21,25}$ In thin films,

16 pair-breaking effect by interstitial Fe is suspected to be much stronger due to its low dimension. It is

17 postulated that the excess Fe in our films, although very small, probably reacts with oxygen to form

18 non-magnetic oxides during the sequential annealing, in a similar way to that found in another

19 report $^{25}$. The increase of $J_{\mathrm{c}}$ may also due to the phase change induced by interstitial oxygen. Similar

20 oxygen annealing studies in $\mathrm{Fe}_{1.08} \mathrm{Se}_{0.45} \mathrm{Te}_{0.55}$ single crystals ${ }^{29}$ and $\mathrm{Fe}_{1+y} \mathrm{Te}_{\mathrm{e}}$ thin films ${ }^{30}$ have been

21 reported, where improved superconductivity emerges with the formation of the new phase after

22 oxygen annealing. It is also possible that both mechanisms are effective at the same time. We have

23 investigated the annealed films extensively through structural characterizations. Our TEM 
examination did not shown any iron oxides. No observable phase change, like Hu et al had seen in

$2 \mathrm{Fe}_{1.08} \mathrm{Se}_{0.45} \mathrm{Te}_{0.55}$ single crystals, can be observed in our films either. It could be due to the very small

3 amount of the excess Fe in our films, since our films were grown from stoichiometric $\mathrm{FeSe}_{0.45} \mathrm{Te}_{0.55}$

4 targets without intentionally introducing excess Fe.

We note that the $T_{\mathrm{c}}$ in our FST films was slightly suppressed after oxygen annealing. This result is different from what happened in oxygen annealed single crystals, where an enhanced $T_{\mathrm{c}}$ was

7 reported. ${ }^{19,23-25}$ The suppression of $T_{\mathrm{C}}$ in our FST films is found in both oxygen and vacuum annealing

8 process. One possible explanation is that the fast cooling after the film growth causes the lattice

9 inhomogeneity in the film and creates imbalanced local strain. It has been reported by Bellingeri et

$10 a l^{19,31,32}$ that the compressive strain in $a b$ plane can enhance the $T_{\mathrm{c}}$ of FST films. In this scenario,

11 the inhomogeneity in the film may lead to a distribution of the strain field, and hence $T_{\mathrm{c}}$. The film

12 may comprise of higher $T_{\mathrm{c}}$ regions with compressive strain and lower $T_{\mathrm{c}}$ regions with tensile strain.

13 The inhomogeneity in the as-grown films results in a relatively wider superconducting transition as

14 shown in the insets of Fig. 1(a) and Fig. 2(a). One of the consequences of the sequential annealing,

15 either in oxygen or vacuum, is the release of strain in the FST films, leading to more homogenous

16 strain distribution. This is consistent with the sharper superconducting transition and slightly

17 lowered on-set $T_{\mathrm{c}}$ observed in the annealed samples.

18 Considering the fact that the $J_{\mathrm{c}}$ of the films increases significantly at an annealing temperature

19 as low as $90^{\circ} \mathrm{C}$ with the presence of oxygen, one could expect a qualitatively similar $J_{\mathrm{c}}$ enhancement

20 by aging in the air at room temperature. Oxygen in the air should slowly react with the freshly grown

21 films. This was indeed the case when we measured the $J_{\mathrm{c}}$ of the fully oxygen-annealed films and the

22 as-grown films after one year stored in a dry environment, but in contact with the air at room

23 temperature. The results of the field dependent $J_{\mathrm{c}}$ before and after one year aging are shown in Fig. 3a. 
1 For the as-grown film, the self-field $J_{\mathrm{c}}$ increases from $0.96 \mathrm{MA} / \mathrm{cm}^{2}$ to $1.53 \mathrm{MA} / \mathrm{cm}^{2}$ and the in-field $J_{\mathrm{c}}$

2 increases by $90 \% \sim 100 \%$ from $2 \mathrm{~T}$ to $4.5 \mathrm{~T}$, in qualitative agreement with the oxygen annealing. For

3 the fully oxygen annealed film, the one-year aging produce virtually no effect - the field dependent $J_{\mathrm{c}}$

4 difference is less than $5 \%$. This observation is in line with both explanations mentioned previously.

5 The oxygen in the air can react with the as-grown film, leading to excess Fe removal or the phase

6 change, but have no effect on fully oxygen annealed films.

For a comprehensive understanding, we also conduct a 2-hour vacuum annealing on a fully oxygen annealed film in order to check the irreversibility of the oxygen annealing effect. The result

9 is shown in Fig. 3b, where black squires, red triangles and blue open circles stand for the field

10 dependent $J_{\mathrm{c}}$ of the film before annealing, after fully oxygen annealing and after an additional

11 vacuum annealing, respectively. It is seen that the vacuum annealing shows virtually no influence

12 on $J_{\mathrm{c}}$ after the film was fully oxygen annealed. The $J_{\mathrm{c}}$ enhancement caused by the oxygen is 13 irreversible.

Fig. 4 shows the direct transport $J_{\mathrm{c}}$ of an oxygen annealed FST film as a function of magnetic field at different temperatures, together with the magnetization-derived $J_{\mathrm{c}}$ at $T=5 \mathrm{~K}$ (solid

16 circles in Fig. 3). The transport measurement was taken after the magnetization measurement by 17 patterning the same film into micro bridges, $300 \mu \mathrm{m}$ long and $10 \mu \mathrm{m}$ wide. $J_{\mathrm{c}}$ was obtained from I-V 18 curves by using $1 \mu \mathrm{V}$ criteria, and a typical one measured at $5 \mathrm{~K}$ under $0.1 \mathrm{~T}$ field is shown in the 19 lower inset of Fig.4. We note the $J_{\mathrm{c}}$ values obtained by the transport measurement and 20 magnetization measurement are virtually the same. Upper inset shows the superconducting 21 transition at $0,1,3,5,9$ Tesla by the resistance measurement. The zero resistance $T_{\mathrm{c}}$ is $17 \mathrm{~K}$ at zero 22 field and $14.5 \mathrm{~K}$ at 9T, applied perpendicular to the film surface.

Figs. 5(a) and 5(b) present the data of the in-field (B//c) $J_{\mathrm{c}}$ and the pinning force, $F_{\mathrm{p}}(=J \times B)$ 
1 in fully oxygen annealed films at $T=5 \mathrm{~K}$, together with the FST coated conductor grown on the

2 rolling-assisted biaxially textured substrate (RABiTS) ${ }^{12}$ and several prominent superconducting

3 materials (4.2 K data), including the second-generation (2G) $\mathrm{YBa}_{2} \mathrm{Cu}_{3} \mathrm{O}_{7}$ (YBCO) wire ${ }^{33,34}$,

4 thermo-mechanically processed $\mathrm{Nb}_{47 \mathrm{Ti}^{35,36}}$ and small grain $\mathrm{Nb}_{3} \mathrm{Sn}$ wire ${ }^{37,38}$. The solid lines in Fig.

5 5(b) are plotted using Kramer's scaling approximations ${ }^{39}$. In general, $J_{\mathrm{c}}$ and $F_{\mathrm{p}}$ of the FST films are

6 higher than that of the Nb-Ti wires, but substantially lower than the 2G YBCO coated conductors. It is

7 interesting to compare the present results with the FST coated conductors. The oxygen annealed films

8 have higher $J_{\mathrm{c}}$ and $F_{\mathrm{p}}$ at low fields, but lower $J_{\mathrm{c}}$ and $F_{\mathrm{p}}$ at high fields. Although we do not fully

9 understand the pinning mechanism in the FST coated conductors at present, a higher performance

10 FST coated conductor may be anticipated when its strong pinning at high field is combined with the

11 enhanced pinning achieved at low field by the practical and cost-efficient oxygen annealing, which

12 doubles the self-field $J_{\mathrm{c}}$. The dotted line in Fig. 5 shows an estimated $J_{\mathrm{c}}$ value we can expect by

13 applying a lifting factor, derived from the magnitude of the $J_{\mathrm{c}}$ enhancement obtained through the

14 oxygen annealing, to the $J_{\mathrm{c}}$ value known for the as-grown FST coated conductor on RABiTS. The

15 proposed optimally annealed FST coated conductor would have a comparable performance with the

$16 \mathrm{Nb}_{3} \mathrm{Sn}$ below 4T, and much better above, and that would represent a significant step forward in the

17 potential high field applications of the iron based superconductors at liquid helium temperature.

In summary, short time ( 1-2 hours) oxygen annealing at low temperature (below $100^{\circ} \mathrm{C}$ ) has been found to be very effective on the $J_{\mathrm{c}}$ enhancement in the FST superconducting thin films.

20 The fully annealed FST thin films exhibit a doubled $J_{\mathrm{c}}$ at self-field and four-fold $J_{\mathrm{c}}$ enhancement

21 above 1.5T. Possible explanations for the observed $J_{\mathrm{c}}$ enhancement in FST films could be the reaction

22 between the excess Fe and oxygen, or the phase change due to the interstitial oxygen - both were

23 previously found effective to improve the superconductivity in the form of single crystals. 
1 Qualitatively, similar $J_{\mathrm{c}}$ enhancement is also achieved by room temperature aging in the air, which is

2 likely due to the same underline mechanism with the oxygen annealing. The reaction between the

$3 \quad$ FST film and oxygen is found irreversible. The oxygen annealing process identified in this study can

4 serve as a fast, easy, and low-cost approach to effectively enhance the $J_{\mathrm{c}}$ in the iron based

5 superconductors. Using this process, the highest self-field $J_{\mathrm{c}}$ of $3 \mathrm{MA} / \mathrm{cm}^{2}$ at $5 \mathrm{~K}$ was achieved in the

6 iron chalcogenides. Combined with other growth induced pinning defects, or artificial pinning

7 centers, such as these produced by the proton or ion irradiation, iron chalcogenide coated conductors

8 are very attractive for the high field application at liquid helium temperature.

9

This work was supported by the U.S. Department of Energy, Office of Basic Energy Science,

11 Division of Materials Science and Engineering, under Contract No. DE-SC0012704. 
Y. Kamihara, H. Hiramatsu, M. Hirano, R. Kawamura, H. Yanagi, T. Kamiya, and H. Hosono. J. Am. Chem. Soc. 128, 10012 (2006).

Y. Kamihara, T. Watanabe, M. Hirano, and H. Hosono. J. Am. Chem. Soc. 130, 3296 (2008).

J. W. Lynn and P. Dai. Physica C: Supercond. 469, 469 (2009).

F.-C. Hsu, J.-Y. Luo, K.-W. Yeh, T.-K. Chen, T.-W. Huang, P. M. Wu, Y.-C. Lee, Y.-L. Huang, Y.-Y. Chu, D.-C. Yan, and M.-K. Wu. Proc. Natl. Acad. Sci. USA 105, 14262 (2008).

M. H. Fang, H. M. Pham, B. Qian, T. J. Liu, E. K. Vehstedt, Y. Liu, L. Spinu, and Z. Q. Mao. Phys. Rev. B 78, 224503 (2008).

T. J. Liu, J. Hu, B. Qian, D. Fobes, Z. Q. Mao, W. Bao, M. Reehuis, S. a. J. Kimber, K. Prokeš, S. Matas, D. N. Argyriou, A. Hiess, A. Rotaru, H. Pham, L. Spinu, Y. Qiu, V. Thampy, A. T. Savici, J. A. Rodriguez, and C. Broholm. Nat. Mat. 9, 718 (2010).

Q. Li, W. Si, and I. K. Dimitrov. Rep. Prog. Phys. 74, 124510 (2011).

J.-F. Ge, Z.-L. Liu, C. Liu, C.-L. Gao, D. Qian, Q.-K. Xue, Y. Liu, and J.-F. Jia. Nat. Mat. 14, 285 (2015).

S. Lee, J. Jiang, Y. Zhang, C. W. Bark, J. D. Weiss, C. Tarantini, C. T. Nelson, H. W. Jang, C. M. Folkman, S. H. Baek, A. Polyanskii, D. Abraimov, A. Yamamoto, J. W. Park, X. Q. Pan, E. E. Hellstrom, D. C. Larbalestier, and C. B. Eom. Nat. Mat. 9, 397 (2010).

F. Kurth, C. Tarantini, V. Grinenko, J. Hänisch, J. Jaroszynski, E. Reich, Y. Mori, A. Sakagami, T. Kawaguchi, J. Engelmann, L. Schultz, B. Holzapfel, H. Ikuta, R. Hühne, and K. Iida. Appl. Phys. Lett. 106, 072602 (2015).

A. Sakagami, T. Kawaguchi, M. Tabuchi, T. Ujihara, Y. Takeda, and H. Ikuta. Physica C: Supercond. 494, 181 (2013). 
W. Si, S. J. Han, X. Shi, S. N. Ehrlich, J. Jaroszynski, A. Goyal, and Q. Li. Nat. Commun. 4, 1347 (2013).

Y. Sun, Y. Tsuchiya, S. Pyon, T. Tamegai, C. Zhang, T. Ozaki, and Q. Li. Supercond. Sci. Tech. 28, 015010 (2015).

P. Yuan, Z. Xu, Y. Ma, Y. Sun, and T. Tamegai. Supercond. Sci. Tech. 29, 035013 (2016).

D. Samal and P. S. Anil Kumar. J. Appl. Phys. 109, 07E129 (2011).

Y. A. Izyumov, Y. N. Proshin, and M. G. Khusainov. Phys. Usp. 172, 113 (2002).

T. M. Mcqueen, Q. Huang, V. Ksenofontov, C. Felser, Q. Xu, H. Zandbergen, Y. S. Hor, J. Allred, A. J. Williams, D. Qu, J. Checkelsky, N. P. Ong, and R. J. Cava. Phys. Rev. B 79, 014522 (2009).

8 Y. Mizuguchi, K. Deguchi, S. Tsuda, T. Yamaguchi, and Y. Takano. Phys. Rev. B 81, 214510 (2010).

19 T. Noji, T. Suzuki, H. Abe, T. Adachi, M. Kato, and Y. Koike. J. Phys. Soc. Jpn. 79, 084711 (2010).

K. Deguchi, Y. Mizuguchi, Y. Kawasaki, T. Ozaki, S. Tsuda, T. Yamaguchi, and Y. Takano. Supercond. Sci. Tech. 24, 055008 (2011).

Y. Mizuguchi, K. Deguchi, Y. Kawasaki, T. Ozaki, M. Nagao, S. Tsuda, T. Yamaguchi, and Y. Takano. J. Appl. Phys. 109, 013914 (2011).

K. Deguchi, D. Sato, M. Sugimoto, H. Hara, Y. Kawasaki, S. Demura, T. Watanabe, S. J. Denholme, H. Okazaki, T. Ozaki, T. Yamaguchi, H. Takeya, T. Soga, M. Tomita, and Y. Takano. Supercond. Sci. Tech. 25, 084025 (2012).

Y. Kawasaki, K. Deguchi, S. Demura, T. Watanabe, H. Okazaki, T. Ozaki, T. Yamaguchi, H. Takeya, and Y. Takano. Solid State Commun. 152, 1135 (2012). 
Y. Sun, T. Taen, Y. Tsuchiya, Z. X. Shi, and T. Tamegai. Supercond. Sci. Tech. 26, 015015 (2013).

Y. Sun, Y. Tsuchiya, T. Taen, T. Yamada, S. Pyon, A. Sugimoto, T. Ekino, Z. Shi, and T. Tamegai. Sci. Rep. 4, 4585 (2014).

L. Zhi, P. Jun-Ping, Z. Hui-Min, Z. Wen-Hao, D. Hao, D. Peng, C. Kai, S. Can-Li, J. Shuai-Hua, W. Lili, H. Ke, C. Xi, X. Qi-Kun, and M. Xu-Cun. J. Phys. Condens. Matter 26, 265002 (2014).

W. Si, J. Zhou, Q. Jie, I. Dimitrov, V. Solovyov, P. D. Johnson, J. Jaroszynski, V. Matias, C. Sheehan, and Q. Li. Appl. Phys. Lett. 98, 262509 (2011).

C. P. Bean. Rev. Mod. Phys. 36, 31 (1964).

H. Hu, J.-M. Zuo, M. Zheng, J. N. Eckstein, W. K. Park, L. H. Greene, J. Wen, Z. Xu, Z. Lin, Q. Li, and G. Gu. Phys. Rev. B 85, 064504 (2012).

H. Hu, J.-H. Kwon, M. Zheng, C. Zhang, L. H. Greene, J. N. Eckstein, and J.-M. Zuo. Phys. Rev. B 90, 180504 (2014).

31 E. Bellingeri, S. Kawale, V. Braccini, R. Buzio, A. Gerbi, A. Martinelli, M. Putti, I. Pallecchi, G. Balestrino, A. Tebano, and C. Ferdeghini. Supercond. Sci. and Tech. 25, 084022 (2012). E. Bellingeri, I. Pallecchi, R. Buzio, A. Gerbi, D. Marrè, M. R. Cimberle, M. Tropeano, M. Putti, A. Palenzona, S. Kaciulis, and C. Ferdeghini. J. Supercond. Nov. Magn. 24, 35 (2010). A. Xu, J. J. Jaroszynski, F. Kametani, Z. Chen, D. C. Larbalestier, Y. L. Viouchkov, Y. Chen, Y. Xie, and V. Selvamanickam. Supercond. Sci. Tech. 23, 014003 (2010).

Z. Chen, F. Kametani, Y. Chen, Y. Xie, V. Selvamanickam, and D. C. Larbalestier. Supercond. Sci. Tech. 22, 055013 (2009).

D. C. Larbalestier and A. W. West. Acta Mater. 32, 1871 (1984). 
$1 \quad 36$

$2 \quad 37$

$3 \quad 38$

$4 \quad 39$

5

L. D. Cooley, P. J. Lee, and D. C. Larbalestier. Phys. Rev. B 53, 6638 (1996).

R. M. Scanlan, W. A. Fietz, and E. F. Koch. J. Appl. Phys. 46, 2244 (1975).

A. Godeke. Supercond. Scie. Tech. 19, R68 (2006).

E. J. Kramer. J. Appl. Phys. 44, 1360 (1973). 
1 Figures and Legends

2

Figure 1

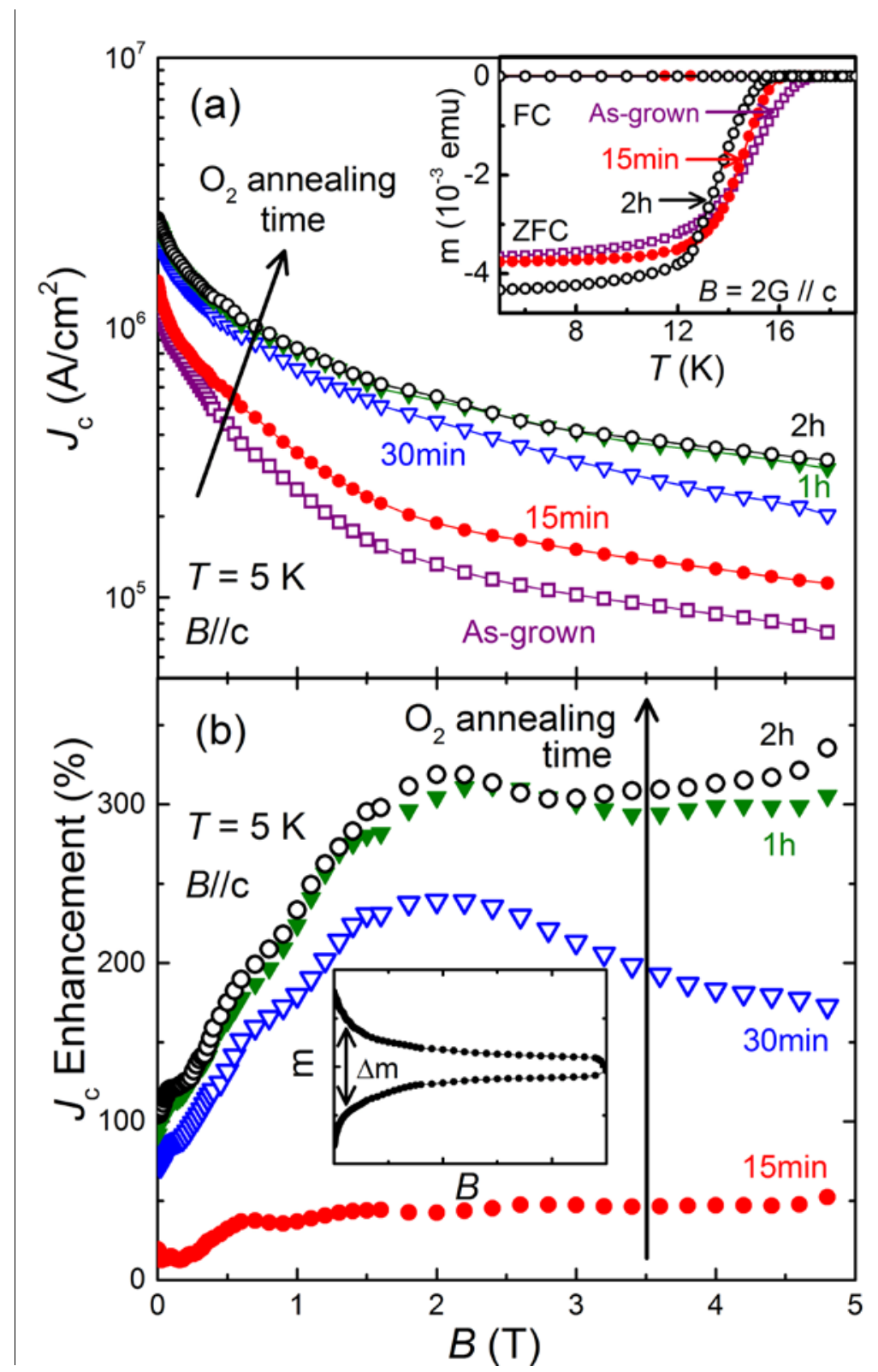

4

Figure 1: Magnetic field dependence of $J_{\mathrm{C}}$ (a) and $J_{\mathrm{c}}$ enhancement (b) of one of the sequentially annealed FST films in 100mTorr oxygen environment for different annealing time. Inset in (a) shows the $T_{\mathrm{c}}$ change by the magnetization measurement of the film under zero field cool (ZFC) and field cool (FC). Inset in (b) shows a typical half magnetization loop from which the field dependent magnetization $J_{\mathrm{c}}$ is derived. 


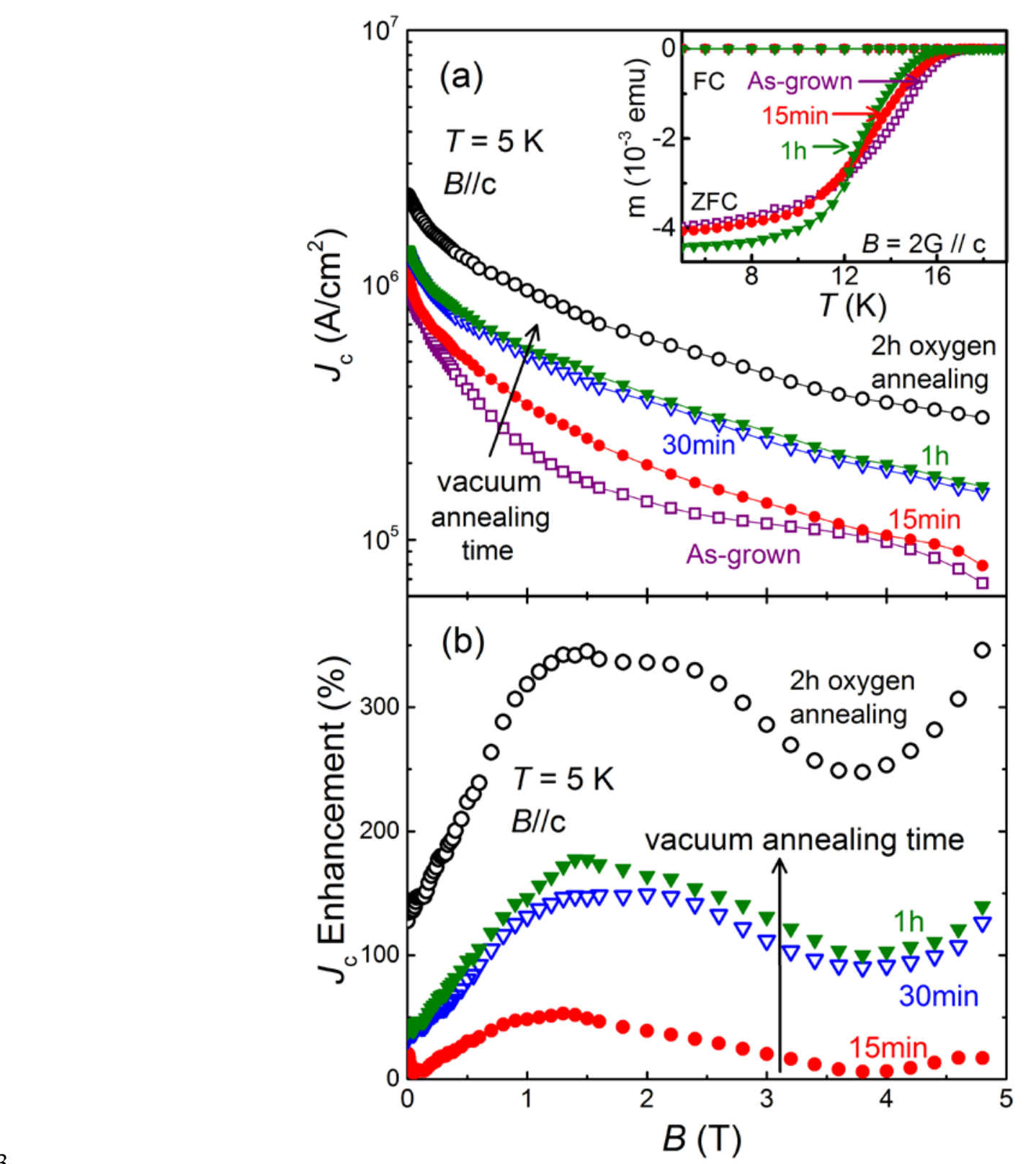

3

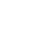

Figure 2: Magnetic field dependence of $J_{\mathrm{c}}$ (a) and $J_{\mathrm{c}}$ enhancement (b) of one of the sequentially vacuum annealed FST films. Inset shows the $T_{\mathrm{C}}$ changes by the magnetization measurement of the film under zero field cool (ZFC) and field cool (FC). The result of the additional two-hour oxygen annealing at $90^{\circ} \mathrm{C}$ following the vacuum annealing is also plotted for comparison (open circles). 

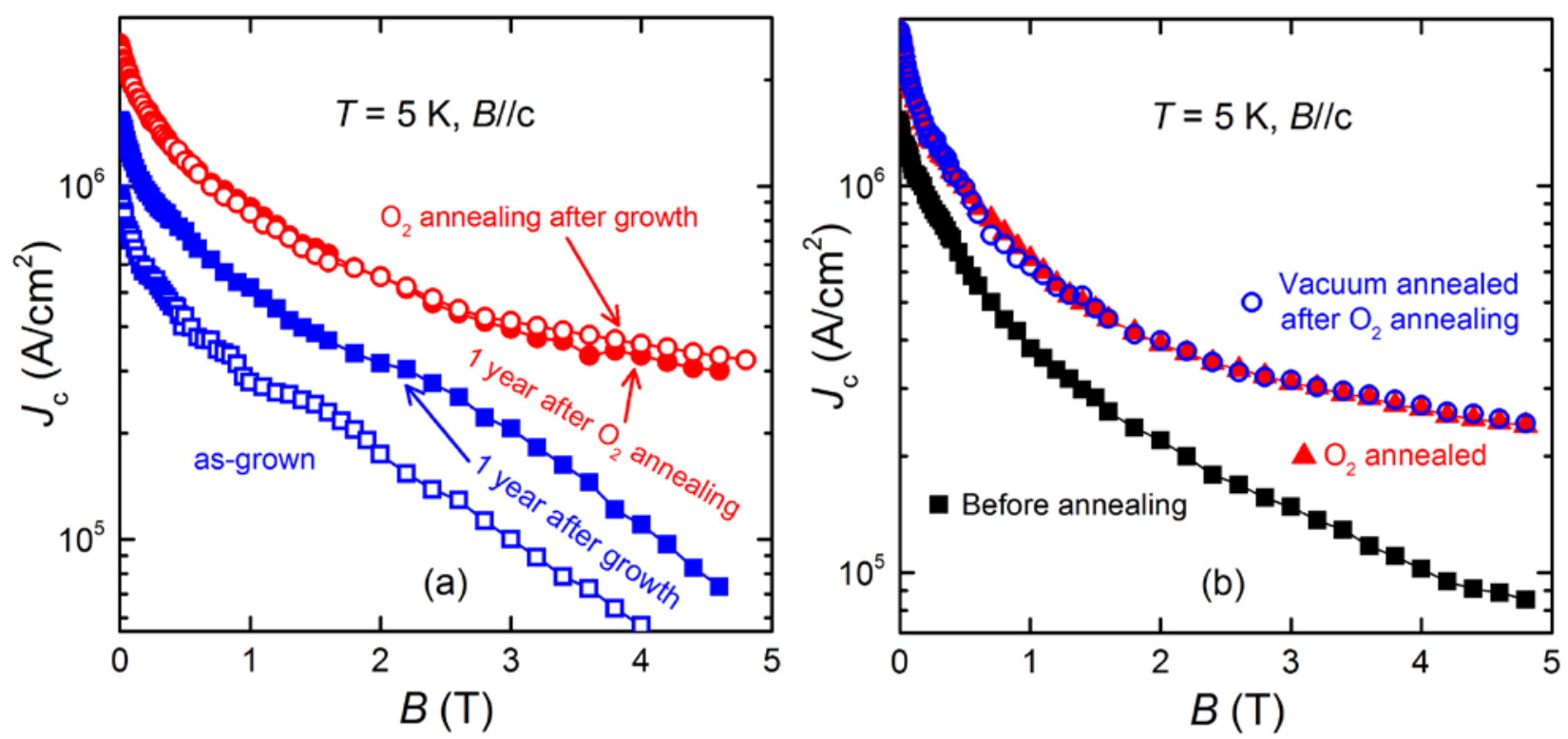

3 Figure 3: (a) Magnetic field dependence of $J_{\mathrm{c}}$ of the FST films upon aging for one year. $J_{\mathrm{c}}$ increases

4 in the as-grown film, while no change in the fully oxygen annealed film. (b) Magnetic field

5 dependent $J_{\mathrm{c}}$ of a FST film which was first fully oxygen annealed and then vacuum annealed. The

6 additional vacuum annealing have virtually no further influence on $J_{\mathrm{c}}$, indicating that the $J_{\mathrm{c}}$

7 enhancing process caused by oxygen is irreversible. 


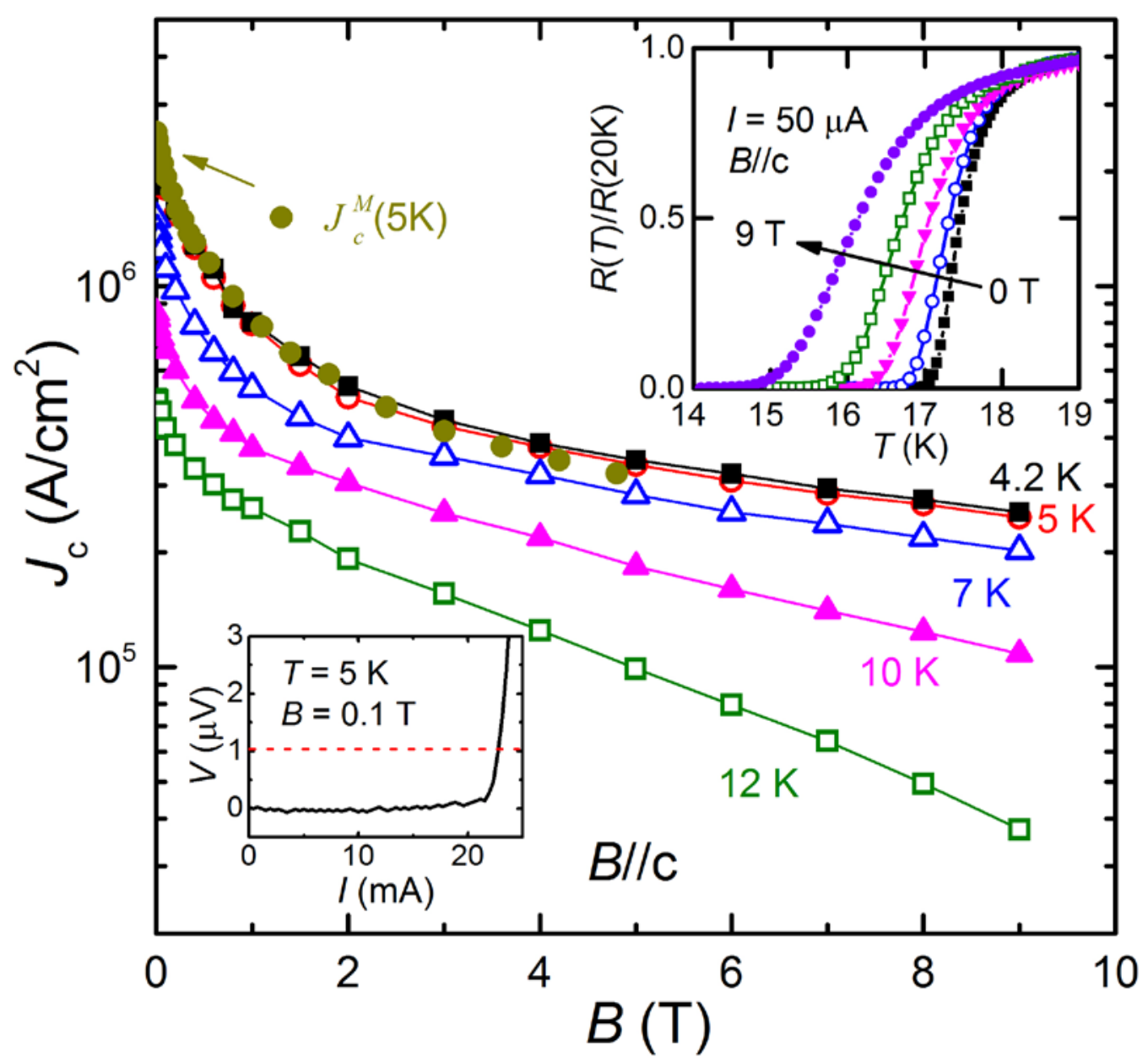

3 Figure 4: Magnetic field dependence of $J_{\mathrm{c}}$ of a fully oxygen annealed FST film at different

4 temperatures by transport measurement. $J_{\mathrm{c}}$ (solid circles) obtained from magnetic hysteresis

5 measurement of the same film at $T=5 \mathrm{~K}$ is also plotted for comparison. Upper inset shows the

6 superconducting transition at various fields (0, 1, 3, 5, 9 Tesla) by the resistive measurement. Lower

7 inset shows a typical $\mathrm{I}-\mathrm{V}$ curve from which the critical current density was extracted using $1 \mu \mathrm{V}$

8 criteria. 


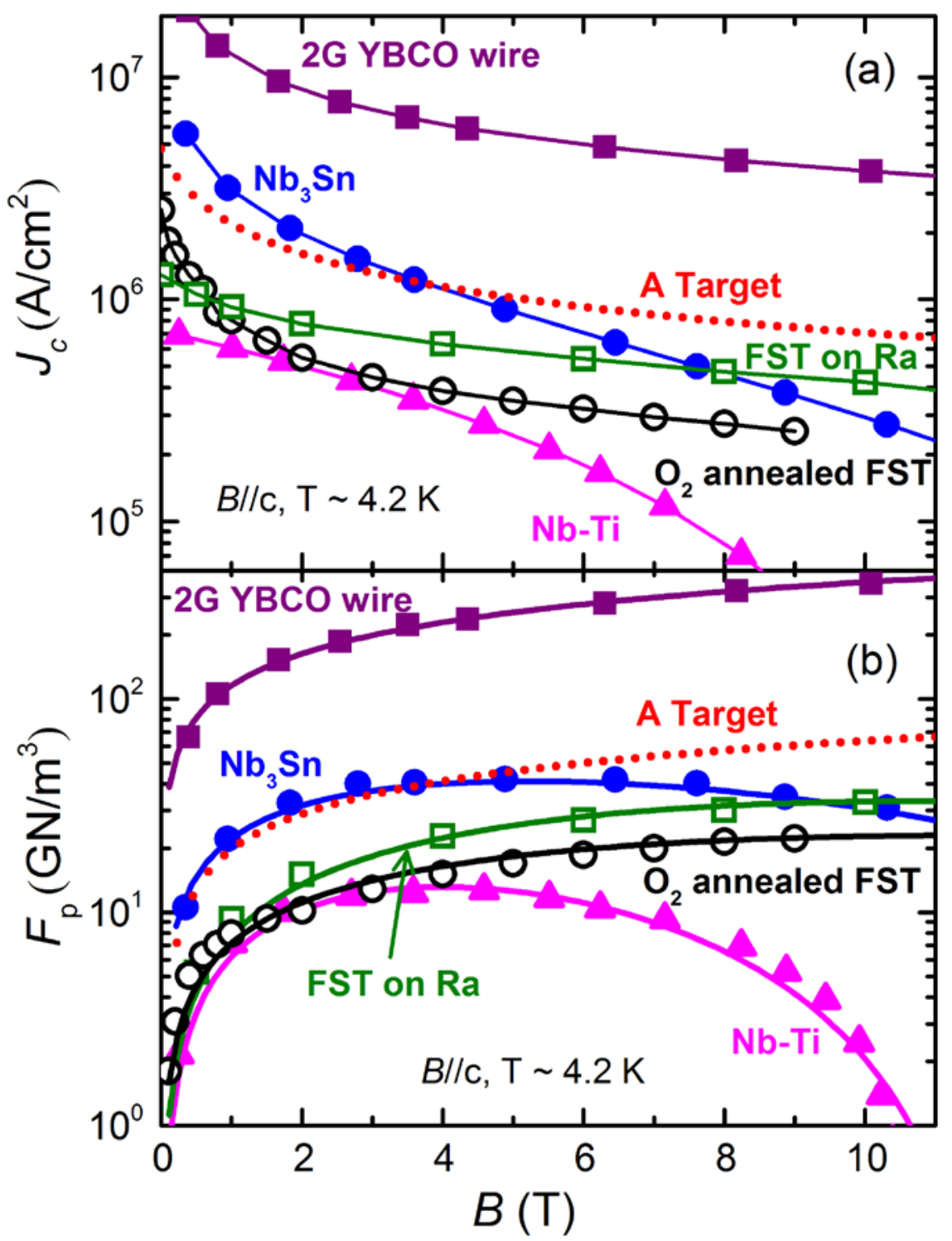

3 Figure 5: Magnetic field dependence of $J_{\mathrm{c}}(\mathrm{a})$ and vortex pining force $F_{\mathrm{p}}(\mathrm{b})$ of several high field 4 superconductors, including oxygen annealed FST film (this work), the FST coated conductors on 5 RABiTS (labeled as "Ra”) ${ }^{12}, \mathrm{Nb}-\mathrm{Ti}, \mathrm{Nb}_{3} \mathrm{Sn}$, and 2G YBCO. The lines in (b) are fitting curves using 6 the Kramer's scaling approximation. $4.2 \mathrm{~K} J_{\mathrm{C}}$ data of $\mathrm{YBCO}, \mathrm{Nb}_{3} \mathrm{Sn}$ and $\mathrm{Nb}-\mathrm{Ti}$ are adopted from 7 literatures ${ }^{33-38}$. The red dotted line shows a practical target to achieve in the near future for the FST 8 coated conductors. 